\title{
On the h-Vector of a Lattice Path Matroid
}

\author{
Jay Schweig \\ Department of Mathematics \\ University of Kansas \\ Lawrence, KS 66044 \\ jschweig@math.ku.edu
}

Submitted: Sep 16, 2009; Accepted: Dec 18, 2009; Published: Jan 5, 2010

Mathematics Subject Classification: 05B35, 05E45

\begin{abstract}
Stanley has conjectured that the h-vector of a matroid complex is a pure Mvector. We prove a strengthening of this conjecture for lattice path matroids by constructing a corresponding family of discrete polymatroids.
\end{abstract}

\section{Introduction}

The h-vector of a finite simplicial complex is arguably its most studied invariant. The following conjecture of Stanley [13] has sparked a great deal of research into the h-vectors of matroid complexes (see Hibi [9], Swartz [14, 15], Chari [5], and Hausel and Sturmfels [6]).

Conjecture 1.1. The h-vector of a matroid complex is a pure M-vector (i.e., the degree sequence of a pure monomial order ideal).

Conjecture 1.1 has been proven for cographic matroids by both Merino [1] and Chari (unpublished).

Lattice path matroids, introduced by Bonin, de Mier, and Noy in 3 and studied further in 4], are special transversal matroids whose bases can be viewed as planar lattice paths. Subclasses of such matroids appeared in [1] and [10]. We show that Stanley's conjecture holds for lattice path matroids by constructing an associated family of pure monomial order ideals. We then strengthen our result by showing that these monomial order ideals are in fact discrete polymatroids, in the sense of Herzog and Hibi 7].

\section{Preliminaries}

We assume a basic familiarity with matroid theory (see, for instance, [12]). 
Recall that the $f$-vector of an $(r-1)$-dimensional simplicial complex $\Delta$ is $\left(f_{0}, f_{1}, \ldots, f_{r}\right)$, where $f_{i}$ counts the number of $(i-1)$-dimensional faces of $\Delta$, and that the $h$-vector of $\Delta$ is the sequence $\left(h_{0}, h_{1}, \ldots, h_{r}\right)$, where $\sum_{i=0}^{r} f_{i}(t-1)^{r-i}=\sum_{i=0}^{r} h_{i} t^{r-i}$.

The $\mathrm{f}$ - and h-vectors of a matroid $M$ are those of its independence complex (the simplicial complex whose faces are the independent sets of $M)$. The h-vector of $M$ is also given by the coefficients of $T(x, 1)$, where $T(x, y)$ is the Tutte polynomial of $M$ (see [2]).

For simplicity, all our matroids will use the ground set $[n+r]$, for positive integers $n$ and $r$. (Here and throughout, $[k]=\{1,2, \ldots, k\}$.) When $B$ is a basis of a matroid $M$, we call $i \in B$ internally active if $(B \backslash i) \cup j$ is not a basis for any $j<i$. The following lemma can be found in 2 .

Lemma 2.1. Let $\left(h_{0}, h_{1}, \ldots, h_{r}\right)$ be the h-vector of a matroid $M$. For $0 \leqslant i \leqslant r, h_{i}$ is the number of bases of $M$ with $r-i$ internally active elements.

A sequence $\left(h_{0}, h_{1}, \ldots, h_{r}\right)$ of nonnegative integers is an $M$-vector if there exists a monomial order ideal $\Gamma$ containing, for all $i, h_{i}$ elements of degree $i$. Some authors, including Stanley in [13], call these objects O-sequences. An M-vector is called pure if there exists a monomial order ideal $\Gamma$ as above with no maximal element of degree less than $r$. Not all M-vectors are pure (for example, $(1,3,1)$ ).

\section{$3 \quad$ Lattice path matroids}

Let $\mathcal{A}=\left\{A_{1}, A_{2}, \ldots, A_{k}\right\}$ be a collection of subsets of $[n+r]$. Recall that a subset $T \subseteq \bigcup_{i=1}^{k} A_{i}$ is a partial transversal of $\mathcal{A}$ if there exists an injection $\phi: T \rightarrow[k]$ such that $t \in A_{\phi(t)}$ for all $t \in T$. The partial transversals of $\mathcal{A}$ are the independent sets of the transversal matroid defined by $\mathcal{A}$ (see [12]), which we denote as $M(\mathcal{A})$.

Definition 3.1. Let $\mathcal{A}=\left\{\left[a_{1}, c_{1}\right],\left[a_{2}, c_{2}\right], \ldots,\left[a_{r}, c_{r}\right]\right\}$, where each $a_{i} \leqslant c_{i}$, each $\left[a_{i}, c_{i}\right]=$ $\left\{a_{i}, a_{i}+1, \ldots, c_{i}\right\}$ is an interval in the integers, $1 \leqslant a_{1}<a_{2}<\cdots<a_{r}$, and $c_{1}<c_{2}<$ $\cdots<c_{r} \leqslant n+r$. Then $M(\mathcal{A})$ is called a lattice path matroid.

For our purposes, a lattice path $\sigma$ to $(n, r)$ is a sequence of unit-length steps, each either directly north or east, beginning at the origin and terminating at $(n, r)$. If $B \subseteq[n+r]$ and $|B|=r$, define a lattice path $\sigma_{B}$ to $(n, r)$ by the following rule: the $i^{\text {th }}$ step of $\sigma$ is north if and only if $i \in B$. Although the path $\sigma_{B}$ depends upon the point $(n, r)$, we suppress this from the notation.

For the remainder of this section, let $\mathcal{A}$ be a collection of sets as in Definition 3.1, let $A=\left\{a_{1}, a_{2}, \ldots, a_{r}\right\}$ and $C=\left\{c_{1}, c_{2}, \ldots, c_{r}\right\}$, and assume all lattice paths terminate at the point $(n, r)$. The following propositions are shown in 3 .

Proposition 3.2. A set $B \subseteq[n+r]$ with $|B|=r$ is a basis of $M(\mathcal{A})$ if and only if the path $\sigma_{B}$ (weakly) lies between the paths $\sigma_{A}$ and $\sigma_{C}$.

Proposition 3.3. Let $B \subseteq[n+r]$ be a basis of $M(\mathcal{A})$. Then $i \in B$ is internally active if and only if the $i^{\text {th }}$ step of $\sigma_{B}$ (which is a north step, by definition) coincides with a north step of $\sigma_{A}$. 
If $\sigma$ is a path between $\sigma_{A}$ and $\sigma_{C}$, call a north step of $\sigma$ tight if it coincides with a north step of $\sigma_{A}$. Otherwise, call it loose. We use these terms only to refer to vertical steps. Lemma 2.1 and Proposition 3.3 immediately imply the following.

Corollary 3.4. Let $\left(h_{0}, h_{1}, \ldots, h_{r}\right)$ be the $h$-vector of $M(\mathcal{A})$. Then for all $i, h_{i}$ is the number of lattice paths between $\sigma_{A}$ and $\sigma_{C}$ that have exactly $i$ loose steps.

Remark 3.5. In general, a rank-r matroid $M$ with coloop e has $\left(h_{0}, h_{1}, \ldots, h_{r-1}, 0\right)$ as its h-vector, where $\left(h_{0}, h_{1}, \ldots, h_{r-1}\right)$ is the h-vector of the deletion $M \backslash e$. Thus, we may restrict our attention to coloop-free matroids. The matroid $M(\mathcal{A})$ is coloop-free if and only if each $a_{i}<c_{i}$.

Theorem 3.6. The h-vector of a lattice path matroid is a pure M-vector.

Proof. Let $\mathcal{A}, A$, and $C$ be as above. We construct a pure monomial order ideal $\Gamma$ whose elements are in bijection with the lattice paths between $\sigma_{A}$ and $\sigma_{C}$ such that the degree of the monomial corresponding to a path $\sigma$ is the number of loose steps of $\sigma$. Given Corollary 3.4, this will prove the theorem.

Let $\sigma$ be a path between $\sigma_{A}$ and $\sigma_{C}$. Define the monomial $m(\sigma)$ as follows: for each loose step of $\sigma$ along the line $x=i$, include a copy of $x_{i}$ in $m(\sigma)$. (See Figure 1.) Let $\Gamma=\left\{m(\sigma): \sigma\right.$ is a path between $\sigma_{A}$ and $\left.\sigma_{C}\right\}$.

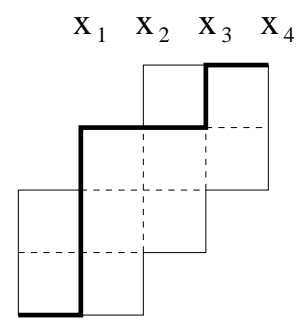

Figure 1: A lattice path $\sigma$ with $m(\sigma)=x_{1}^{2} x_{3}$.

Let $m(\sigma) \in \Gamma$ have degree less than $r$, and suppose that the highest tight step of $\sigma$ is on the line $x=i$. Then the next step of $\sigma$ must be an east step. Letting $\sigma^{\prime}$ be the path obtained from $\sigma$ by changing this last tight step to an east step and following it by a north step, we see that $m\left(\sigma^{\prime}\right)=m(\sigma) x_{i+1}$. By Remark 3.5, $\sigma^{\prime}$ lies between $\sigma_{A}$ and $\sigma_{C}$. Continuing in this way, we eventually obtain a degree- $r$ monomial in $\Gamma$ divisible by $m(\sigma)$, which shows that $\Gamma$ is pure.

To see that the correspondence $\sigma \mapsto m(\sigma)$ is injective, consider $m=x_{i_{1}}^{e_{1}} e_{i_{2}}^{e_{2}} \cdots x_{i_{m}}^{e_{m}} \in \Gamma$ with $i_{1}<i_{2}<\cdots<i_{m}$ and each $e_{j}>0$. We show that there is a unique way to construct a path $\sigma$ between $\sigma_{A}$ and $\sigma_{C}$ such that $m(\sigma)=m$. Start at the point $(n, r)$. First, travel to the line $x=i_{m}$ by taking west steps, except when forced to take south steps by the path $\sigma_{A}$. Second, take all forced tight south steps and then $e_{m}$ loose south steps. Continue this process by traveling to the line $x=i_{m-1}$, and so on. This clearly produces the unique path $\sigma$ with $m(\sigma)=m$. Moreover, if $m=m^{\prime} x_{j}$ for some $j$, simply modify the above algorithm to take one fewer south step along $x=j$. The resulting path corresponds to the monomial $m^{\prime}$, which proves that $\Gamma$ is an order ideal. 


\section{Discrete polymatroids}

In [7, the authors introduce discrete polymatroids, which generalize matroids in the way monomial order ideals generalize simplicial complexes. Further algebraic properties of these objects were studied in [8]. The definition in [7] involves integer vectors, but we treat these sequences as exponents of monomials. All our monomial order ideals use the variables $\left\{x_{i}\right\}$. If $m$ is a monomial, let $m_{i}$ denote the degree of $x_{i}$ in $m$.

Definition 4.1. A monomial order ideal $\Gamma$ is a discrete polymatroid if, whenever $m, m^{\prime} \in$ $\Gamma$ with $\operatorname{deg}(m)>\operatorname{deg}\left(m^{\prime}\right)$, there is some $i$ such that $m_{i}>m_{i}^{\prime}$ and $m^{\prime} x_{i} \in \Gamma$.

The following proposition is shown in [7].

Proposition 4.2. A pure monomial order ideal $\Gamma$ is a discrete polymatroid if and only if, for any two maximal monomials $m, m^{\prime} \in \Gamma$ and index $i$ with $m_{i}>m_{i}^{\prime}$, there exists an index $j$ such that $m_{j}<m_{j}^{\prime}$ and $\frac{x_{j}}{x_{i}} m \in \Gamma$.

If we require that all monomials be squarefree, Definition 4.1 and Proposition 4.2 specialize to classical matroid axioms.

Theorem 4.3. The monomial order ideal $\Gamma$ constructed in the proof of Theorem [3.6 is a discrete polymatroid.

Proof. (We are indebted to Joe Bonin for his significant simplification of our original proof.) Let $\mathcal{A}, A, C$, and $\Gamma$ be as in Theorem 3.6, and set $A^{+}=\left\{a_{1}+1, a_{2}+1, \ldots, a_{r}+1\right\}$. By Remark 3.5, the path $\sigma_{A^{+}}$does not cross the path $\sigma_{C}$. It is clear that the set of degree- $r$ monomials of $\Gamma$ is $\left\{m(\sigma): \sigma\right.$ is a path between $\sigma_{A^{+}}$and $\left.\sigma_{C}\right\}$. We show that these monomials satisfy the condition of Proposition 4.2.

Let $\sigma$ and $\sigma^{\prime}$ be two paths in between $\sigma_{A^{+}}$and $\sigma_{C}$, with $m(\sigma)_{i}>m\left(\sigma^{\prime}\right)_{i}$. We will show that there is some $j$ with $m(\sigma)_{j}<m\left(\sigma^{\prime}\right)_{j}$ and some path $\hat{\sigma}$ in between $\sigma$ and $\sigma^{\prime}$ so that $m(\hat{\sigma})=\frac{x_{j}}{x_{i}} m(\sigma)$. Let $q$ be the greatest integer so that the point $(i, q)$ is on the path $\sigma$, and define $q^{\prime}$ analogously for the path $\sigma^{\prime}$.

First, suppose that $q>q^{\prime}$. Since the paths $\sigma$ and $\sigma^{\prime}$ intersect at their common terminal point, there must be a $j>i$ such that $m(\sigma)_{j}<m\left(\sigma^{\prime}\right)_{j}$. Choose $j$ to be minimal with this property (but still greater than $i$ ). Then every east step of $\sigma$ between the lines $x=i$ and $x=j$ is strictly above an east step of $\sigma^{\prime}$. Let $t$ be the least integer so that $(j, t)$ lies on the path $\sigma$. Define a new path $\hat{\sigma}$ from $\sigma$ as follows: delete the north step going from $(i, q-1)$ to $(i, q)$, and add a new north step going from $(j, t-1)$ to $(j, t)$. Then move the part of $B$ going from $(i, q)$ to $(j, t)$ down a unit step. Since $q>q^{\prime}$ and $j$ was chosen to be minimal, the resulting path $\hat{\sigma}$ is between $\sigma$ and $\sigma^{\prime}$. Because $\hat{\sigma}$ has one more north step than $\sigma$ along the line $x=j$ and one fewer than $\sigma$ along the line $x=i, m(\hat{\sigma})=\frac{x_{j}}{x_{i}} m(\sigma)$.

Next suppose that $q \leqslant q^{\prime}$. Because $m(\sigma)_{i}>m\left(\sigma^{\prime}\right)_{i}, \sigma$ has more north steps along the line $x=i$ than $\sigma^{\prime}$. Rotating everything by 180 degrees, we return to the first case considered.

Definition 4.4. We call a sequence $\left(h_{0}, h_{1}, \ldots, h_{r}\right)$ a PM-vector if it is the degree sequence of some discrete polymatroid $\Gamma$. 
Every PM-vector is a pure M-vector (by definition), but the converse does not hold: the M-vector $(1,4,2)$ is pure but not PM. Thus, the following corollary strengthens Theorem 3.6 .

Corollary 4.5. The h-vector of a lattice path matroid is a PM-vector.

Given the above corollary, it seems natural to ask the following.

Question 4.6. Which matroids have h-vectors that are PM-vectors?

In 11, Merino proves Conjecture 1.1] for cographic matroids. Although the pure monomial order ideals constructed in his proof are rarely discrete polymatroids, we have yet to find a matroid (cographic or otherwise) whose h-vector is not a PM-vector.

Acknowledgements. My thanks go to Joe Bonin and Ed Swartz for many helpful discussions, and to the anonymous referee for numerous edits and suggestions.

\section{References}

[1] F. Ardila, The Catalan matroid, J. Combin. Theory Ser. A 104 (1) (2003), 49-62.

[2] A. Björner, The homology and shellability of matroids and geometric lattices, In Matroid Applications, N. L. White, ed., Cambridge University Press, Cambridge (1992) 226-283.

[3] J. Bonin, A. de Mier, and M. Noy, Lattice path matroids: enumerative aspects and Tutte polynomials, J. Combin. Theory Ser. A 104 (1) (2003), 63-94.

[4] J. Bonin and A. de Mier, Lattice path matroids: structural properties, European J. Combin. 27 (5) (2006), 701-738.

[5] M.K. Chari, Two decompositions in topological combinatorics with applications to matroid complexes, Trans. Amer. Math. Soc. 349 (10) (1997), 3925-3943.

[6] T. Hausel and B. Sturmfels, Toric hyperKähler varieties, Doc. Math. 7 (2002), 495534 (electronic).

[7] J. Herzog and T. Hibi, Discrete polymatroids, J. Algebraic Combin. 16 (3) (2002), 239-268.

[8] J. Herzog, T. Hibi, and M. Vladoiu, Ideals of fiber type and polymatroids, Osaka J. Math. 42 (4) (2005), 807-829.

[9] T. Hibi, What can be said about pure O-sequences? J. Combin. Theory Ser. A 50 (2) (1989), 319-322.

[10] C. Klivans. Shifted Matroid Complexes. PhD thesis, Massachusetts Institute of Technology, 2003.

[11] C. Merino, The Chip Firing Game and Matroid Complexes, in Discrete Models: Combinatorics, Computation, and Geometry, DM-CCG 2001, R. Cori, J. Mazoyer, M. Morvan, and R. Mosseri, eds., Discrete Mathematics and Theoretical Computer Science Proceedings AA (2001), 245-256. 
[12] J. Oxley, Matroid Theory, Oxford University Press, Oxford, 1992.

[13] R. Stanley, Combinatorics and Commutative Algebra, Birkhauser, Boston, MA, 1996.

[14] E. Swartz, g-elements of matroid complexes, J. Combin. Theory Ser. B 88 (2) (2003), 369-375.

[15] E. Swartz, g-elements, finite buildings, and higher Cohen-Macaulay connectivity, $J$. Combin. Theory Ser. A 113 (7) (2006), 1305-1320. 